\title{
Acetylcholine receptor turnover in mice with passively transferred myasthenia gravis II. Receptor synthesis
}

\author{
STEPHEN WILSON, ANGELA VINCENT, JOHN NEWSOM-DAVIS \\ From the Department of Neurological Science, Royal Free Hospital School of Medicine, London, UK
}

SUMMARY The in vivo synthesis of diaphragm endplate acetycholine receptors was estimated in mice treated daily with IgG from eight myasthenia gravis patients. Myasthenia gravis IgG preparations which had previously been shown to increase the rate of receptor degradation also increased the rate of receptor synthesis, suggesting the existence of a compensatory mechanism serving to stabilise the number of acetycholine receptors.

An increased rate of acetylcholine receptor (AChR) degradation is thought to play an important role in the loss of end-plate AChRs which occurs in myasthenia gravis (MG). The rate of loss of ${ }^{125} \mathrm{I}-\mathrm{a}-$ bungarotoxin (a-butx)-labelled AChRs is increased both in myoblast cultures ${ }^{12}$ and in mice injected daily with $\mathrm{IgG}$ prepared from MG patients. ${ }^{3} \mathrm{We}$ have confirmed these results but find that the increased rate of degradation is not an invariable result of human antibodies binding to mouse AChR at the end-plate. ${ }^{4}$ Moreover, even in those animals in which an increased rate of AChR degradation could be demonstrated the total number of acetylcholine receptors present at the end-plate after seven daily IgG injections was reduced less than predicted from the increased degradation rate.

These observations suggested that an increased rate of AChR synthesis might compensate for the increased rate of degradation that occurred with some IgG preparations ${ }^{4}$ and in this paper we describe the results of experiments designed to test this possibility. End-plate AChRs were first blocked by intrathoracic injection of non-radioactive a-butx. The animals then received daily injections of $\mathrm{IgG}$. The rate of synthesis of new AChRs, free from a-butx, was measured at various time intervals by removing the diaphragms and incubating in radioactive a-butx in vitro. AChR synthesis was increased

Address for reprint requests: Dr A Vincent, Royal Free Hospital School of Medicine, Pond St, London NW3 2QG, UK.

Received 15 November 1982

Accepted 9 December 1982 in animals injected with IgG preparations which increased AChR degradation.

\section{Methods}

Patients The IgG preparations were obtained from the ten patients described in the preceding paper. ${ }^{4}$ Mice of both the AKR strain and the B6D2F1 hybrids were again used. The IgG was purified by the Rivanol ammonium sulphate method from plasma obtained during plasma exchange therapy.

Synthesis of junctional AChRs of mouse diaphragm in vivo Groups of animals $(n=10)$ were anaesthetised with halothane as previously described ${ }^{4}$ and a-butx $(0.1 \mu \mathrm{g} / \mathrm{g}$ body weight) was injected into the left hemi-thorax. The amount of a-butx was critical as high doses led to an unacceptable mortality rate, and too low a dose caused insufficient blocking of the end-plate AChR. The exact amount injected was determined from preliminary experiments in which the animals were killed four hours later and their diaphragms incubated in vitro in ${ }^{125} \mathrm{I}$-a-butx as described in the preceding paper. ${ }^{4}$ The amount of a-butx injected was designed to block more than $90 \%$ of the mouse end-plate AChRs without killing the animals. The value for unblocked $A C h R s$ at 4 hours was used as $R_{O}$ (see below).

The injected animals were killed at intervals over 1-9 days and the appearance of AChRs free of a-butx was measured by incubation of the diaphragm in vitro in ${ }^{125} \mathrm{I}-$ a-butx as described previously. ${ }^{4}$ The number of observed AChRs ( $R$ obs) was expressed as a percentage of the initial total number present in diaphragms from untreated mice. The rate of synthesis of AChR was determined using the degradation constants $(K)$ derived from the preceding paper: if $R_{s}$ is the actual number of AChRs synthesised by any particular time $t$, then $R_{s}=T . r$ where $r$ is the frac- 
tional synthesis rate per day. $\mathbf{R}_{\mathrm{Obs}}$, the observed number of AChRs, is made up of $r / K(1-\exp (-K t))$, the number of newly synthesised $A C h R s$ which remain, and $R_{O}$ $\exp (-\mathrm{Kt})$ the number of residual unblocked AChRs $\left(R_{O}\right)$ which are still present (see ref 5).

$$
\begin{aligned}
& \text { Robs }=\frac{r}{K}(1-\exp (-K t))+\text { Ro exp }(-K t) \\
& \text { therefore } r=\frac{(\text { Robs }-\operatorname{Ro} \exp (-K t)) K}{(1-\exp (-K t)} \\
& \text { and Rs }=\frac{(\text { Robs }- \text { Ro exp }(-K t)) K t}{1-\exp (-K t)}
\end{aligned}
$$

The values for $R_{s}$ were calculated and plotted against time and a line drawn through them by the method of linear regression.

The effect of MG and control IgG upon the rate of synthesis of junctional AChRs in vivo was assessed by injecting $15 \mathrm{mg} /$ day of $\mathrm{IgG}$ intraperitoneally into each

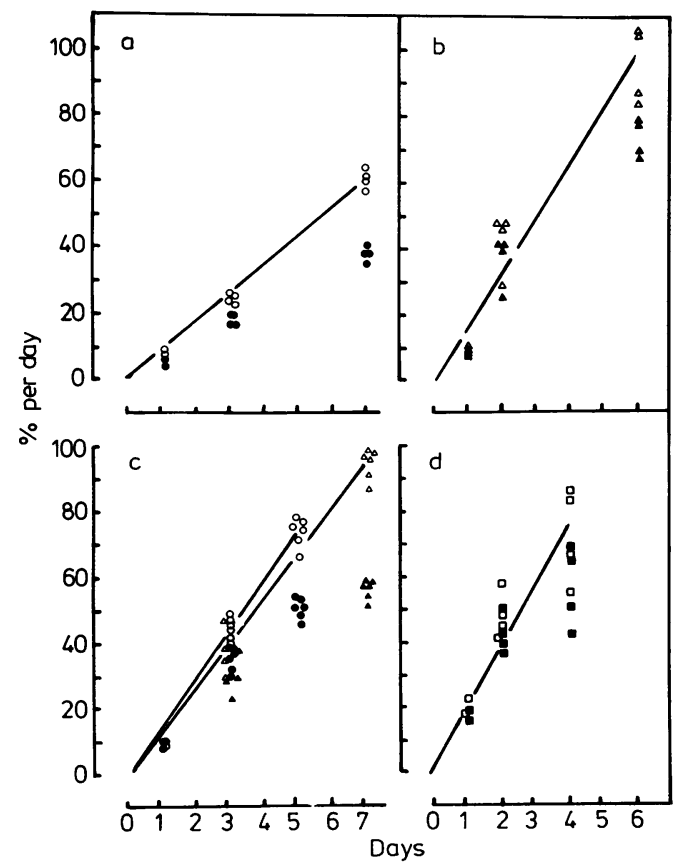

Fig 1 Experiments designed to show AChR synthesis in mice injected with control or MG IgG preparations. In each case the closed symbols represent the experimental observations (Robs) and the open symbols the calculated number of AChRs synthesised (Rs) as a percentage of the total number present initially.

$A \quad A K R$ mice injected with control IgG.

B B6D2F1 injected with $M G 10 \mathrm{IgG}$,

$C A K R(\triangle)$ and $B 6 D 2 F 1(O)$ mice injected with IgG 7,

$D A K R$ mice injected with IgG 4. Note that the observed appearance (Robs) of new binding sites as well as the calculated synthesis (Rs) is increased in these $M G \operatorname{IgG}$ treated animals. animal starting after the intrathoracic injection of a-butx. Cyclophosphamide $(300 \mathrm{mg} / \mathrm{kg})$ was given as a single dose on day 1 to suppress the immunological response to the foreign protein and the preparations of $\mathrm{IgG}$ were the same as those used in the previous study. ${ }^{4}$

\section{Results}

In this study we have taken advantage of the essentially irreversible nature of a-butx binding to block existing AChRs at the mouse diaphragm end-plates by intrathoracic injection, and have measured the appearance of AChRs free from bound a-butx over a period of days by incubation in ${ }^{125} \mathrm{I}-\mathrm{a}$ - butx in vitro. The non-endplate bound ${ }^{125} \mathrm{I}-\mathrm{a}-\mathrm{butx}$, which was subtracted, was always less than $10 \%$ of the control treated-endplate binding and did not differ between control (uninjected), control IgG and MG IgG treated animals.

An example of results from an experiment performed with control IgG is shown in fig $1 \mathrm{~A}$, and from three using MG IgG preparations in fig $1 B, C, D$. The rate of synthesis in control IgG treated animals was similar to that derived from experiments in non-IgG treated animals and with the measured rate of degradation in untreated animals ${ }^{4}$ (see table 1 ). The rate of appearance of newly synthesised AChRs in end-plates of MG IgG treated animals was variable and in some cases did not differ from control values (table 1). In contrast, some preparations, for example, MG 4, 6, 7 and 10 (see fig 1) caused a significant increase in the rate of appearance of unlabelled AChRs.

For each experiment the synthesis rate constant was derived using the equation given in the methods. The effect of MG IgG on the calculated rate of synthesis of $\mathrm{AChR}$ was not apparently related to the clinical state of the patient, to the anti-AChR titre against human or mouse AChR, or to the binding of human antibodies to the mouse $\mathrm{AChR}$ in vivo. However, those IgG preparations which had no effect upon the rate of AChR degradation in the previous experiments ${ }^{4}$ also had no effect on the rate of synthesis determined here, and the IgG preparations which had caused an acceleration of degradation also brought about an increased synthesis of new AChRs. This increased synthesis rate was clearly not due simply to the use of an increased degradation rate in the calculation since the actual observed number of newly synthesised AChRs was also increased (see fig 1). When the results of experiments in both AKR and B6D2F1 mice were combined there was a significant positive correlation between the rates of degradation and synthesis $(p<0.01$; fig 2$)$. 
Table 1 The effect of $\mathrm{IgG}$ upon the rate of degradation and synthesis of AChRs

\begin{tabular}{|c|c|c|c|}
\hline & Patient & $\begin{array}{l}\text { AChR degradation } \\
\text { \%/day } \dagger\end{array}$ & $\begin{array}{l}\text { AChR synthesis } \\
\text { \%/day }\end{array}$ \\
\hline & $\begin{array}{l}\text { Control } 1 \\
\text { Control } 2\end{array}$ & $\frac{9.5}{-} 0.4( \pm S E n=4)$ & $\begin{array}{l}9.4 \pm 0.4(\text { SE } n=4) \\
9.7(n=2)\end{array}$ \\
\hline \multirow[t]{2}{*}{ AKR MICE } & $\begin{array}{ll}\text { MG } & 1 \\
\text { MG } & 2 \\
\text { MG } & 3 \\
\text { MG } & 4 \\
\text { MG } & 5 \\
\text { MG } & 6 \\
\text { MG } & 7 \\
\text { MG } & 8 \\
\text { MG } & 9 \\
\text { MG } & 10\end{array}$ & $\begin{array}{c}8 \cdot 5 \\
10 \cdot 0 \\
9 \cdot 8 \\
11 \cdot 9^{*} \\
9 \cdot 1 \\
15 \cdot 0 \ddagger \\
15 \cdot 7^{*} \ddagger \\
9 \cdot 4 \\
10 \cdot 2 \\
12 \cdot 2^{*}\end{array}$ & $\begin{array}{l}\overline{-} \\
\overline{7} \cdot 8 \\
18 \cdot 2 \ddagger \\
9 \cdot 7 \\
12 \cdot 3^{*} \\
13 \cdot 4 / 14 \cdot 8^{*} \\
\overline{15} \\
15 \cdot 5 \ddagger\end{array}$ \\
\hline & Control 1 & $9.4 \pm 0.4( \pm S E n=4)$ & $9 \cdot 2 \pm 0.2( \pm \operatorname{SE~} n=3)$ \\
\hline B6D2F1 MICE & $\begin{array}{ll}\text { MG } & 1 \\
\text { MG } & 2 \\
\text { MG } & 3 \\
\text { MG } & 4 \\
\text { MG } & 5 \\
\text { MG } & 6 \\
\text { MG } & 7 \\
\text { MG } & 8 \\
\text { MG } & 9 \\
\text { MG } & 10\end{array}$ & $\begin{array}{c}10 \cdot 2 \\
12 \cdot 2^{*} \\
7 \cdot 6 \\
13 \cdot 5 \ddagger \\
9 \cdot 4 \\
9 \cdot 4 \\
19 \cdot 3 \ddagger \\
9 \cdot 8 \\
10 \cdot 1 \\
10 \cdot 8^{*}\end{array}$ & $\begin{array}{c}- \\
- \\
7 \cdot 8 \\
14 \cdot 6 \ddagger \\
9 \cdot 6 \\
10 \cdot 7 \\
15 \cdot 9 \ddagger \\
8 \cdot 4 \\
8 \cdot 5 \\
16 \cdot 7 \ddagger\end{array}$ \\
\hline
\end{tabular}

*p $<0.01, \neq p<0.001$, (Student's $t$ test)

† Results derived from ref 4.

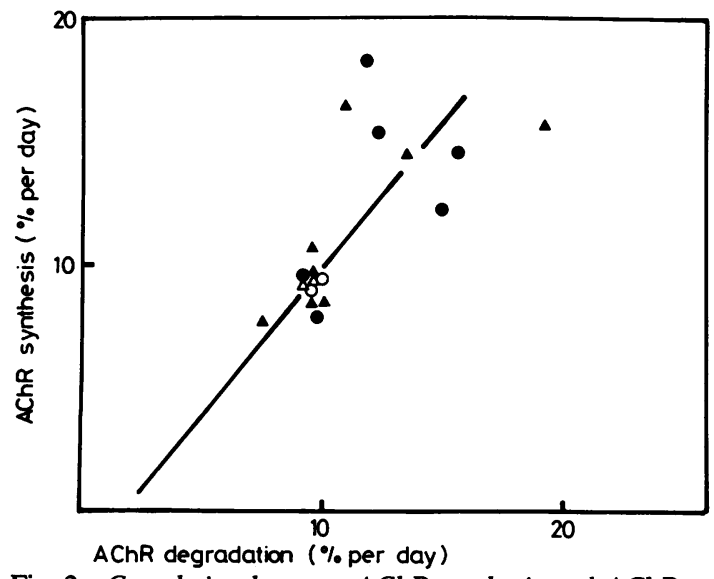

Fig 2 Correlation between AChR synthesis and AChR degradation rates for $A K R$ (circles) and B6D2F1 (triangles) mice.

Open circles are from control IgG treated animals.

\section{Discussion}

This method for estimating the rate of synthesis of end-plate acetylcholine receptors in vivo has not been described previously and depends on certain assumptions. First, the rate of dissociation of unlabelled a-butx from the AChRs in vivo must be relatively small and not affected by the injected IgG preparations. The rate of dissociation of toxin under culture conditions at $37^{\circ} \mathrm{C}$ has previously been demonstrated for mouse and rat muscle by Heinemann $e t a l^{6}$ and Reiness $e t a l^{7}$ who inhibited AChR degradation with dinitrophenol ${ }^{6}$ or sodium cyanide. ${ }^{7}$ They obtained rate constants for dissociation of a-butx of about $4 \%$ per day. Under similar conditions we found a value of less than $2 \%$ per day. ${ }^{4}$ If this value is taken into account in the loss of endplate a-butx binding, the values obtained (in percent/day) for both degradation and synthesis would be lower, but the relative increase in degradation or synthesis in MG IgG treated animals would in fact be a little higher. We found no evidence that MG IgG preparations could by a direct effect increase the rate of dissociation of a-butx. ${ }^{4}$

The calculation of Rs, the actual number of AChRs synthesised, depends critically on the rate of degradation. Since degradation experiments could not be performed on the same animals as the synthesis experiments, this could be a source of error. The calculation also assumes that newly synthesised AChRs are degraded at the same rate as those labelled with ${ }^{125} \mathrm{I}-\mathrm{a}$-butx in the degradation experiments. This is difficult to prove but the apparent first order kinetics for loss of ${ }^{125} \mathrm{I}$-a-butx from mammalian endplates observed by ourselves and others in degradation experiments ${ }^{67}$ suggests the existence of a single turnover rate rather than a mixture of different rates. 
One further factor is that the end-plate could become functionally inactive as a result of injection of the almost saturating amounts of a-butx combined with MG IgG; this might affect the rate of degradation and synthesis of the receptors which is now known to increase when end-plates are experimentally denervated by cutting the nerve. ${ }^{89}$ However there was no evidence of any denervation effect as judged by the lack of extra-junctional ${ }^{125} \mathrm{I}$-a-butx binding in both these experiments and those reported in the preceding paper. ${ }^{4}$

The rate of synthesis of acetylcholine receptors found in uninjected control animals was very similar to that determined in mice injected with control IgG or with IgG from MG patients that did not increase degradation. However the rate of $\mathrm{AChR}$ synthesis was increased in those animals in which an increased rate of degradation had previously been found. ${ }^{4}$ The increased appearance of newly synthesised AChRs was evident even before the correction for calculation of the true rate of synthesis, which included the degradation rate constant, had been applied. This indicates that the increased rate of synthesis was not due merely to the use of a larger degradation constant in the calculations. There was a significant correlation between the rates of degradation and rates of synthesis in animals treated with the individual MG IgG preparations.

The extra-junctional AChRs which appear after denervation or during in vitro culture, show a rate of synthesis which is controlled independently of the fractional degradation rate which is constant. ${ }^{5}$ One previous study found no increase in the synthesis of AChRs on cultured myoblasts during the six hours immediately after MG IgG had been removed. ${ }^{10} \mathrm{On}$ the other hand, evidence of increased AChR synthesis was found in chick myoblast cultures between 6 and 10 hours after MG IgG treatment. ${ }^{11}$ As far as the intact neuromuscular junction is concerned synthesis and degradation may not be independent. The increased fractional degradation rate which has recently been described for endplate AChRs after denervation of the mouse diaphragm ${ }^{8}$ appears to be balanced by an increased synthesis since the total number of endplate AChR declines only slowly after denervation.

Our findings may have important implications for the mechanisms of AChR receptor loss in MG. The fact that human anti-AChR antibody can bind to mouse AChR without affecting either the rate of degradation or the rate of synthesis may be at least partly due to cross-reactivity between the species and may not indicate that these patients' antibodies fail to degrade their own receptors in vivo. However, the rate of synthesis of AChRs was consistently increased in animals treated with those MG preparations which also increased degradation. This finding suggests the existence of a compensatory mechanism at the mouse post-synaptic membrane that serves to stablilise the number of AChRs. A similar mechanism existing in human MG muscle would tend to limit the overall loss of acetylcholine receptor.

Any effects of MG anti-AChR antibodies in humans, however, would be complicated by the existence of other mechanisms of functional AChR loss. For instance, MG patients have humoral factors that directly block pharmacological activation of the AChR in vivo. ${ }^{12} 13$ More importantly, there is ample evidence that complement plays a major role in loss of AChR containing post-synaptic membrane ${ }^{1415}$ and it is not possible at present to determine the rates of degradation or synthesis in human endplates which have undergone morphological changes. However such experiments have been performed on rats with experimentally induced myasthenia gravis. AChR incorporation into damaged endplates appeared to be reduced, probably due to lack of available post synaptic membrane. ${ }^{15}$ Therefore if a compensatory increase in synthesis does occur in human MG endplates, its relevance may be restricted to those at which structural damage is not too severe.

We thank the Muscular Dystrophy Association of Great Britain and the Medical Research Council for support.

\section{References}

${ }^{1}$ Anwyl R, Appel SH, Narahashi T. Myasthenia gravis serum reduces acetylcholine sensitivity in cultured rat myotubes. Nature 1977;267:262-3.

${ }^{2}$ Bevan S, Kulberg RW, Heinemann S. Human myasthenic sera reduce acetylcholine sensitivity of human muscle cells in tissue culture. Nature 1977;267:263-5.

${ }^{3}$ Stanley EF, Drachman DB. Effect of myasthenic immunoglobulin on acetylcholine receptors of intact neuromuscular junctions. Science 1978;200:1285-6.

${ }^{4}$ Wilson S, Vincent A, Newsom-Davis J. Acetylcholine receptor turnover in mice with passively transferred myasthenia gravis. I. Receptor degradation. J Neurol Neurosurg Psychiatry 1983;46:377-382.

${ }^{5}$ Linden DC, Fambrough DM. Biosynthesis and degradation of aceteylcholine receptors in rat skeletal muscles. Effects of electrical stimulation. Neuroscience 1974;4:527-38.

${ }^{6}$ Reiness CG, Weinberg CB, Hall ZW. Antibodies to acetylcholine receptor increases degradation of junctional and extra-junctional receptors in adult muscle. Nature 1978;274:68-70.

${ }^{7}$ Heinemann S, Bevan S, Kullberg R, Lindstrom J, Rice J. 
Modulation of acetylcholine receptor by antibody against the receptor. Proc Natl Acad Sci 1977;74:3090-4.

${ }^{8}$ Levitt TA, Salpeter MM. Denervated endplates have a dual population of junctional acetylcholine receptors. Nature 1981;291:239-41.

9 Stanley EF, Drachman DB. Denervation accelerates the degradation of junctional acetycholine receptors. Exp Neurol 1981;73:390-6.

${ }^{10}$ Drachman DB, Angus CW, Adams RN, Kao I. Effect of myasthenic patients' immunoglobulin on acetylcholine receptor turnover: Selectivity of degradation process. Proc Natl Acad Sci 1978;75:3422-6.

${ }^{11}$ Fulpius BW, Miskin R, Reich E. Antibodies from myasthenic patients that compete with cholinergic agents for binding to nicotinic receptors. Proc Natl Acad Sci 1980;77:4326-30.

${ }^{12}$ Ito Y, Miledi R, Molenaar PC, Newsom-Davis J, Polak RL, Vincent A. Neuromuscular transmission in myasthenia gravis and the significance of anti-acetylcholine receptor antibodies. In: Lunt GG, Marchbanks RM, eds. Biochemistry of myasthenia gravis and muscular dystrophy. London: Academic Press, 1978;89-110.

${ }^{13}$ Lerrick AJ, Wray D, Vincent A, Newsom-Davis J. Electrophysiological effects of serum factors in myasthenia gravis studied in mouse diaphragm. Ann Neurol 1982 (in press).

${ }^{14}$ Sahashi K, Engel AG, Lambert EH, Howard FM. Ultrastructural localisation of the terminal and lytic 9th complement component (C9) at the motor endplate in myasthenia gravis. J Neurol Exp Neuropathol 1980;39:160-72.

${ }^{15}$ Engel AG, Fumagalli G. Mechanisms of acetylcholine receptor loss from the neuromuscular junction. In: Evered D, ed. Receptors, antibodies and disease. Ciba Foundation Symposium 90; 1982:197-224; Pitman Press. 